\title{
Redécouverte de deux dépôts vendéens du Bronze moyen 2, Petosse et " Massigny »
}

Rediscovering the Middle Bronze Age hoards from Petosse and Massigny in

Vendée

Muriel Mélin

\section{CpenEdition}

\section{Journals}

Édition électronique

URL : https://journals.openedition.org/rao/5926

DOI : $10.4000 /$ rao. 5926

ISSN : $1775-3732$

Éditeur

Presses universitaires de Rennes

Édition imprimée

Date de publication : 18 décembre 2020

Pagination : 61-74

ISBN : 978-2-7535-8232-3

ISSN : 0767-709X

\section{Référence électronique}

Muriel Mélin, "Redécouverte de deux dépôts vendéens du Bronze moyen 2, Petosse et « Massigny » »,

Revue archéologique de l'Ouest [En ligne], 36 | 2020, mis en ligne le 18 décembre 2020, consulté le 22 août 2022. URL : http://journals.openedition.org/rao/5926 ; DOI : https://doi.org/10.4000/rao.5926

Tous droits réservés 


\title{
Redécouverte de deux dépôts vendéens du Bronze moyen 2, Petosse et "Massigny»
}

\section{Rediscovering the Middle Bronze Age hoards from Petosse and Massigny in Vendée}

\author{
Muriel MÉLIN $^{a}$
}

\begin{abstract}
Résumé : Deux dépôts métalliques vendéens composés de haches du Bronze moyen 2 sont réétudiés dans cet article. Découverts au cours de la deuxième moitié du $\mathrm{XIX}^{\mathrm{e}}$ s., ces dépôts étaient depuis longtemps considérés comme disparus. Les haches qui les composaient ont pourtant été pour partie retrouvées au musée archéologique départemental de Nantes. Leur examen permet d'abord de corriger les mélanges faits entre ces deux dépôts, mais aussi de rediscuter certaines attributions typochronologiques. On est en mesure de rétablir ici la composition originelle du dépôt dit de "Massigny " (trouvé en fait sur la commune de Chaix), et d'une partie de l'important dépôt de Petosse, ceci grâce à la patine caractéristique et heureusement préservée de ses haches.
\end{abstract}

\begin{abstract}
Two metal hoards found in western France (Vendée) composed of axes dating from the Middle Bronze Age 2 are the object of a new study in this paper. Discovered during the second half of the 19th century, the hoards have long been considered lost. However, some of the axes have been found in the archaeological museum of Nantes among artefacts labelled without provenance. A new examination has provided the opportunity to correct mix up between the two hoards in the initial publication, and to discuss their chronological attribution. We will present the original compositions of the "Massigny" hoard (actually found in Chaix) and an important part of the Petosse hoard using the characteristic and fortunately preserved patina of the objects.
\end{abstract}

Mots clés : dépôt métallique, âge du Bronze moyen 2, haches à rebords, haches à talon.

Keywords: metal hoard, Middle Bronze Age, flanged axes, palstaves.

\section{REDÉCOUVERTE DES OBJETS}

Ces deux ensembles ont été découverts au cours de la deuxième moitié du XIX ${ }^{\mathrm{e}} \mathrm{s}$. Ils sont tous deux composés uniquement de haches, et ont été enfouis au cours du Bronze moyen 2. Ils ont encore en commun de regrouper principalement des haches à rebords de type vendéen. Les deux, enfin, ont été disposés dans des vases. L’un est cependant constitué de seulement trois haches (" Massigny »), tandis que l'autre regroupe plus de 60 haches. Ils étaient situés à quelques kilomètres l'un de l'autre, à l'ouest de Fontenayle-Comte (fig. 1). Le paysage environnant est par ailleurs très différent puisque celui de "Massigny " était situé sur un point haut, naturellement fortifié et surplombant la rivière
Vendée, alors que l'ensemble de Petosse a été découvert au milieu d'un champ de plaine.

Si ces deux importants dépôts ont été publiés anciennement, décrits et en partie dessinés, ils ont en effet été considérés comme perdus pendant de nombreuses années, les travaux de l'abbé $\mathrm{H}$. Breuil devenant ainsi l'unique source de leur étude (Pautreau, 1979; Gomez de Soto, 1995). Ces dépôts ont souffert de n’avoir pu être étudiés depuis 1902, année de la publication la plus complète que l'on ait pour ces ensembles, écrite par l'abbé Breuil.

Dans le cadre de l'étude d'un ensemble métallique tout à fait comparable, découvert en 2009 à Mouilleron-en-Pareds dans le même département (Mélin, 2011; 2012; 2014), nous avons eu l'occasion de nous pencher à nouveau sur

a Service départemental d'archéologie du Morbihan, chercheuse associée UMR 6566 CReAAH (muriel.melin@hotmail.fr) 

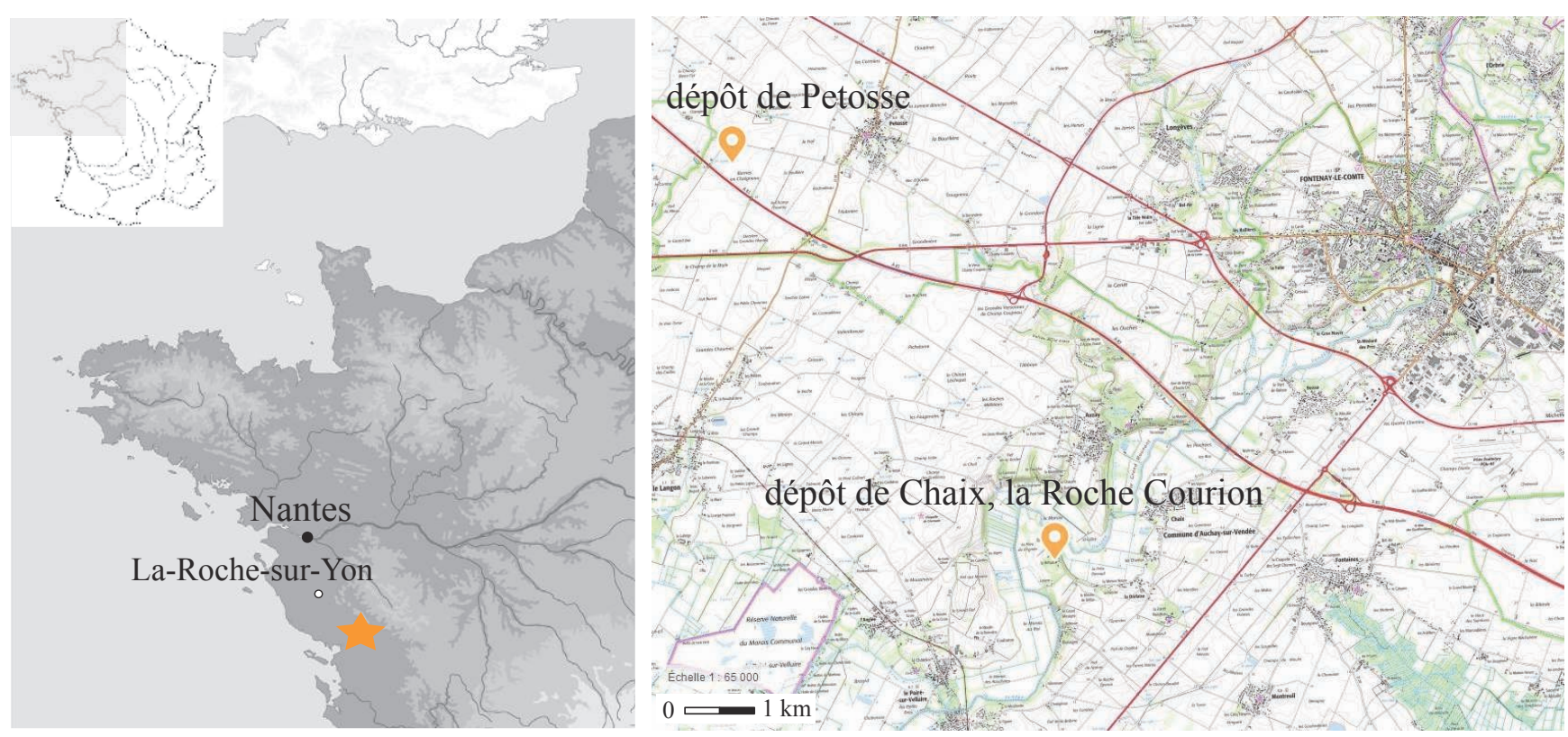

Figure 1 : Carte de localisation des dépôts de Petosse et de la Roche Courion à Chaix (dit de "Massigny ") en Vendée : ils sont à faible distance l'un de l'autre (fond de carte IGN). La localisation pour le dépôt de Petosse est cependant approximative.

Figure 1: Location of the Petosse hoard and the La Roche Courion hoard at Chaix (also referred to as from "Massigny") in Vendée. They were found at a short distance from one another even if the precise location of the Petosse hoard is not known.

les dépôts de Petosse et " Massigny " à travers la littérature ancienne d'une part, mais également à l'appui des objets eux-mêmes. Ceux-ci séjournent en effet pour partie dans les réserves du musée archéologique départemental de Nantes, le musée Dobrée. Les haches de "Massigny » et Petosse ont en effet fait partie des collections d'Octave de Rochebrune, dont son fils Raoul de Rochebrune hérita, avant de les léguer au musée archéologique en 1924 (Parpoil, 2007). C’est grâce au récolement effectué par l'équipe du musée Dobrée que certaines pièces décorées ont été retrouvées et se sont vues réattribuer une provenance, ce grâce à une mise en parallèle avec les dessins de l'abbé Breuil.

Plusieurs haches ont ainsi retrouvé une provenance, en particulier donc, les haches à talon et les haches à rebords décorées qui sont figurées par Breuil. Mais c'est ensuite par leur patine très caractéristique et heureusement préservée de la restauration (pour presque toutes) que de nombreuses autres pièces désignées comme sans provenance peuvent selon nous également être rattachées à ces ensembles.

Grâce à un retour sur les mentions et figurations anciennes de ces découvertes, conjointement à l'observation des pièces elles-mêmes, nous pouvons proposer ici :

- une correction des attributions de provenance faites par H. Breuil (confusion entre les deux dépôts) pour certaines pièces;

- une reconstitution de la composition originelle de ces dépôts (bien qu'encore incomplète pour celui de Petosse);
- mais également une correction d'attribution typochronologique (pour la hache dite à butée médiane de "Massigny").

Des zones d'ombre subsistent pour le dépôt de Petosse principalement, en ce qui concerne le nombre exact de haches, la localisation exacte de sa découverte et la conservation actuelle de plusieurs haches non retrouvées... Revenons d'abord sur les découvertes de chacun de ces ensembles, avant d'exposer les erreurs d'attribution entre ces deux dépôts et les corrections que nous pouvons proposer.

\section{Le dépôt de Chaix, la Roche Courion (OU « MASSIGNY »)}

\section{Circonstances de la découverte}

C'est en 1891 que l'ensemble dit de "Massigny " a été découvert. Voici les informations que nous livre O. de Rochebrune en 1901 : «Le 2 avril 1891, Pierre L'Amiral et Cadet, terrassiers, employés à tirer du caillou pour empierrer les routes, sur un plateau élevé (la cote de l'état-major est de $21 \mathrm{~m}$ ) qui domine tout le pays comme un grand tumulus situé au sud-est de Brillac, non loin du village de Massigny, mirent au jour un vase en terre grossière, très épais, mal cuit, dont la pâte jaune et noire était mêlée d'une grande quantité de parcelles minuscules de silex, et pétrie à la main 

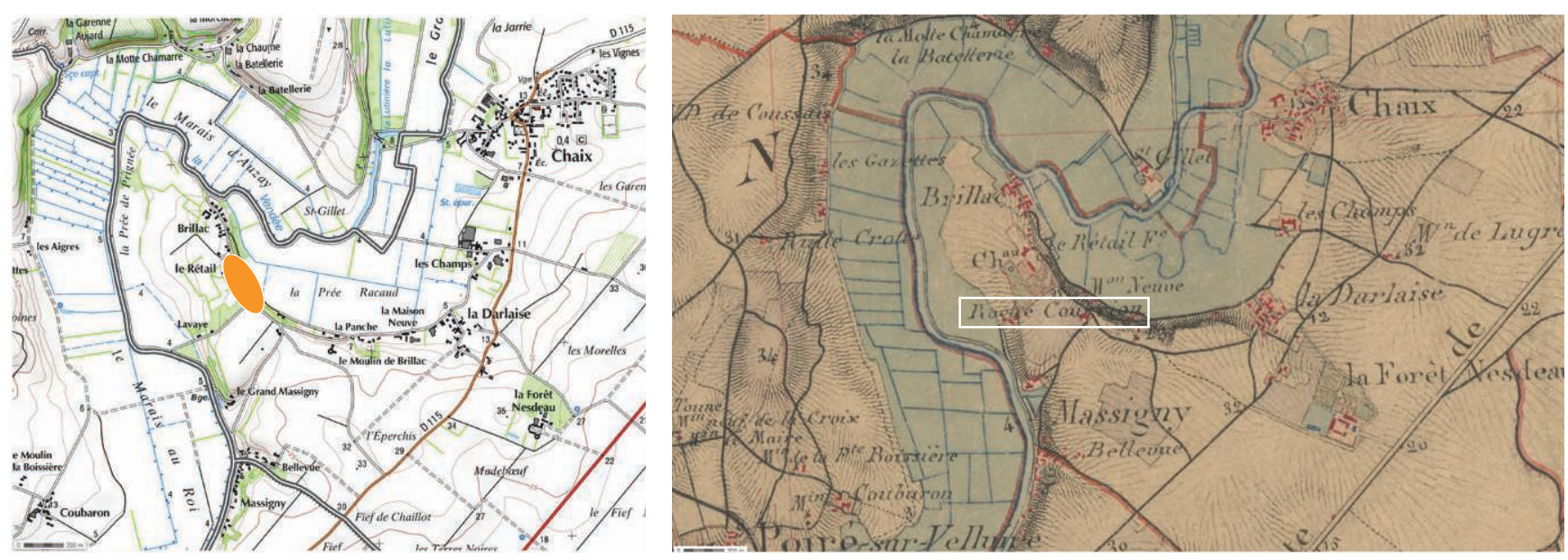

Figure 2 : Localisation de la découverte du dépôt dit de "Massigny ", en fait trouvé à la Roche Courion (toponyme disparu) sur la commune de Chaix, Vendée (fond de carte État-major et IGN avec limites de commune surlignées).

Figure 2: Location of the la Roche Courion hoard at Chaix (toponym no longer exists) (19th century and present day maps with parish limits highlighted).

sans l'usage du tour. Ce vase [...] avait $16 \mathrm{~cm}$ de diamètre au fond sur $25 \mathrm{~cm}$ de hauteur. [...] Il contenait trois haches en bronze placées debout dans ce récipient qui reposait luimême sur le fond, à $40 \mathrm{~cm}$ de profondeur dans le sol. »

\section{Localisation de la découverte : Chaix et non Massigny}

D'après les informations livrées ici, le dépôt provient d'un "plateau élevé $[. .$.$] au sud-est de Brillac, non loin du$ village de Massigny ». O. de Rochebrune précise plus loin: " de Massigny ou de la Roche-Courion, point celtique placé juste à côté " (p. 278), et ensuite : "[les haches] enfouies près de la Roche-Courion ». Ce toponyme a aujourd'hui disparu sur les cartes de l'IGN, mais il est visible sur la carte de l'État-major de 1846, dont parle de Rochebrune. La cote 21 est effectivement visible, juste au-dessus de "Roche-Courion" (fig. 2). Il semble donc bien, comme l'avait déjà replacé B. Poissonnier (1997, p. 96), que le dépôt se trouvait près de la Roche Courion et par conséquent sur la commune de Chaix, et non celle de Velluire, dont dépend Massigny. Nous devrions donc plutôt parler du dépôt de Chaix, la Roche Courion ${ }^{1}$ que du dépôt de Massigny tel qu'il est connu dans la littérature archéologique actuelle à la suite de l'abbé Breuil (1902b, p. 930), qui reprendra fidèlement les indications de provenance fournies par O. de Rochebrune.

Les trois haches ont été trouvées dans un vase dont la facture, de pauvre qualité, est bien décrite par de Rochebrune.

1. Pour distinguer ces haches des haches plates de Chaix (Pautreau, 1979).
Elles se trouvaient en position verticale, détail intéressant que l'on retrouve dans d'autres dépôts contemporains comme ceux fouillés récemment à Mouilleron-en-Pareds (Vendée) (Mélin, 2012) et Saint-Germain-d'Esteuil (Gironde) (Lagarde-Cardona, 2012) par exemple.

Dans son article, O. de Rochebrune compare les haches de Chaix à celles du dépôt trouvé à Petosse trente ans auparavant. Sur la base de cette comparaison et du dessin de deux haches qu'il donne, on peut penser que les trois haches étaient du même type, à rebords - il n'aurait sans doute pas manqué de préciser si l'une d'elles était à talon -, et que les trois étaient vraisemblablement de type vendéen, portant qui plus est des ornementations, courantes sur ce type, faites de cannelures sur les plats et d'un martelage décoratif sur les profils. O. de Rochebrune parle bien de haches « fondues dans le même moule et martelées de la même façon" (1880, p. 278).

\section{Le dépôt de Petosse}

\section{Circonstances de la découverte}

La première mention de ce dépôt, également acquis par O. de Rochebrune, remonte à 1880 ; celui-ci décrit une découverte faite vers 1860, dont la localisation n'est pas plus précise qu' " entre Mouzeuil et Petosse » (fig. 3).

"Nous vîmes entrer un paysan, ayant sur ces épaules un bissac [...] qui paraissait lourdement chargé. Quelle ne fut pas notre surprise, lorsqu'il en vida le contenu, de voir apparaitre sous nos yeux une suite de soixante et quelques haches celtiques de bronze, recouvertes de la terre rouge sous 

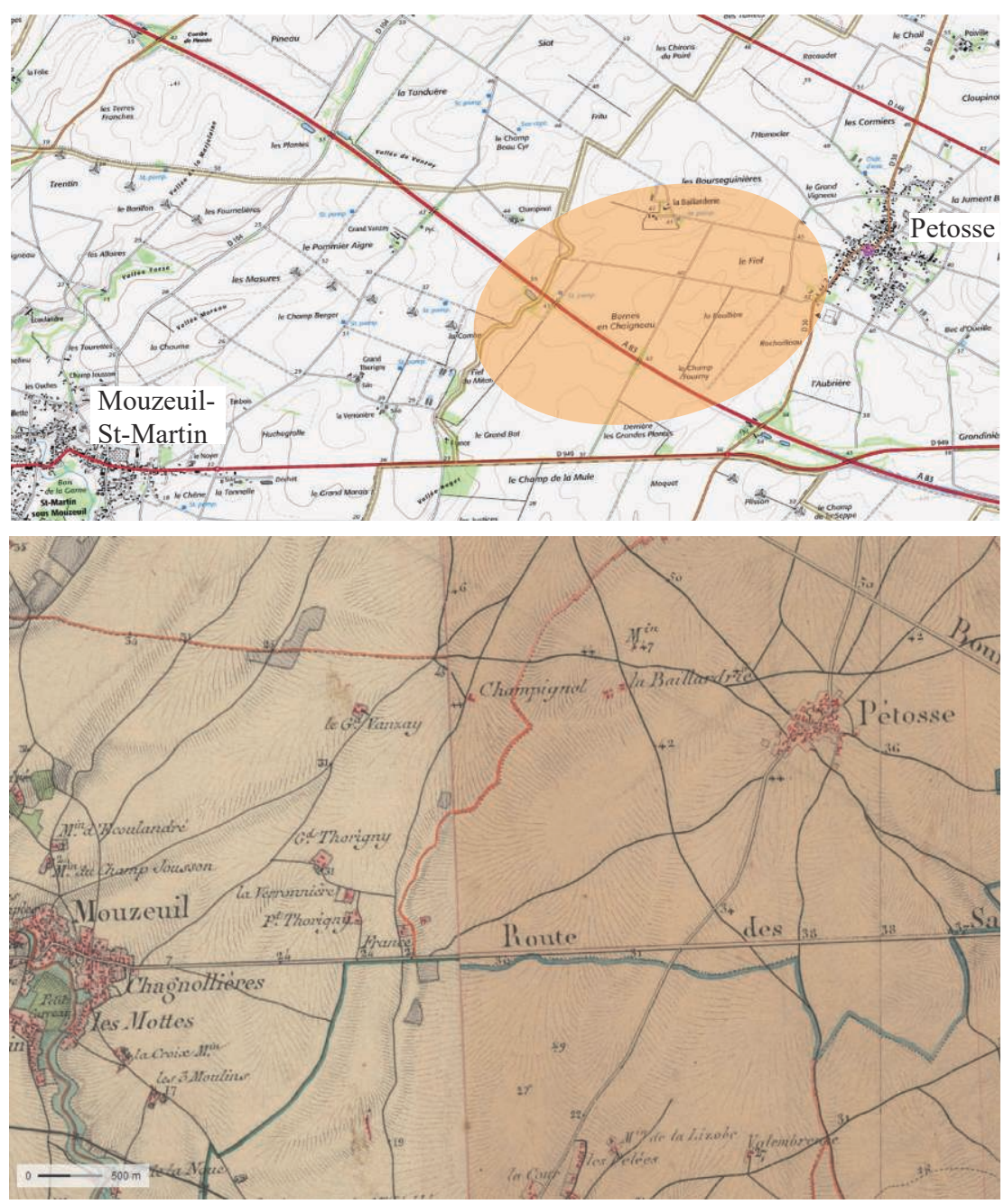

Figure 3 : Localisation approximative de la découverte de Petosse (Vendée), exactement : dans la plaine " entre Mouzeuil et Petosse " (fond de carte État-major et IGN). Figure 3: Approximate location of the Petosse hoard found in the plain "between Mouzeuil and Petosse" (19th century and present day maps). laquelle elles avaient été enfouies pendant des siècles! Ces haches étaient peu variées de formes; elles n'offraient que sept ou huit types divers [...]. J'achetai le tout au poids : il y en avait environ soixante livres pesant. L'inventeur me raconta qu'il les avait découvertes dans la plaine qui s'étend entre Mouzeuil et Petosse, au milieu d'un champ, à une petite profondeur. Elles étaient rangées avec beaucoup de soin dans un vase carré en terre rouge, qu'il n'eut pas la présence d'esprit de conserver, mais dont il apportait quelques débris. » [note de bas de page : "Ce vase était grossièrement fabriqué à la main; la terre était cuite et remplie de fragments siliceux. »] (Rochebrune, 1880)².

2. Des confusions ont été faites quant à la commune de provenance de ce lot. En effet, dans son catalogue manuscrit daté de 1917, le fils d'Octave, Raoul, reprenant vraisemblablement les écrits de son père, fait part de la découverte de 62 haches à Nalliers : il s'agirait du dépôt de Petosse selon B. Poissonnier (1997, p. 200). En outre, elles ont aussi été attribuées pendant un temps à Saint-Étienne-de-Brillouet : c'est ce que publie dans son premier article l'abbé Breuil, avant de se corriger dans son deuxième. Il suit apparemment les indications erronées de G. de Fontaines,
Autre indication que l'on peut noter dans son texte très descriptif et d'une grande qualité : O. de Rochebrune a enlevé "avec soin l'argile fixée à chacun des celts ", sans nettoyage plus poussé. C'est ainsi que des restes de sédiment sont heureusement encore observables aujourd'hui sur les haches; la présence de ce sédiment est d'ailleurs un bon critère de reconnaissance des haches issues de ce dépôt. O. de Rochebrune évoque par ailleurs, concernant la facture de ces haches, la grande habilité de l'artisan (qui « devait être un artiste réputé de l'époque ») et le grand soin apporté au traitement de ces objets après leur fonte, ce qui lui fait dire que « chacun de ces celts devait valoir un ou deux moutons au moins ». Parmi ces haches, il précise qu'un seul spécimen est " brut de fonte", avec de fortes bavures : il est figuré sur sa planche de 1880 à gauche du no 10 (ici fig. 4) mais aussi au $\mathrm{n}^{\circ} 1$ de la planche de 1901 (ici fig. 5).

qui avait lui-même étiqueté deux haches parvenues dans sa collection : "Saint-Étienne-de-Brillouet " (étiquettes encore présentes sur les haches comme nous avons pu l'observer). 


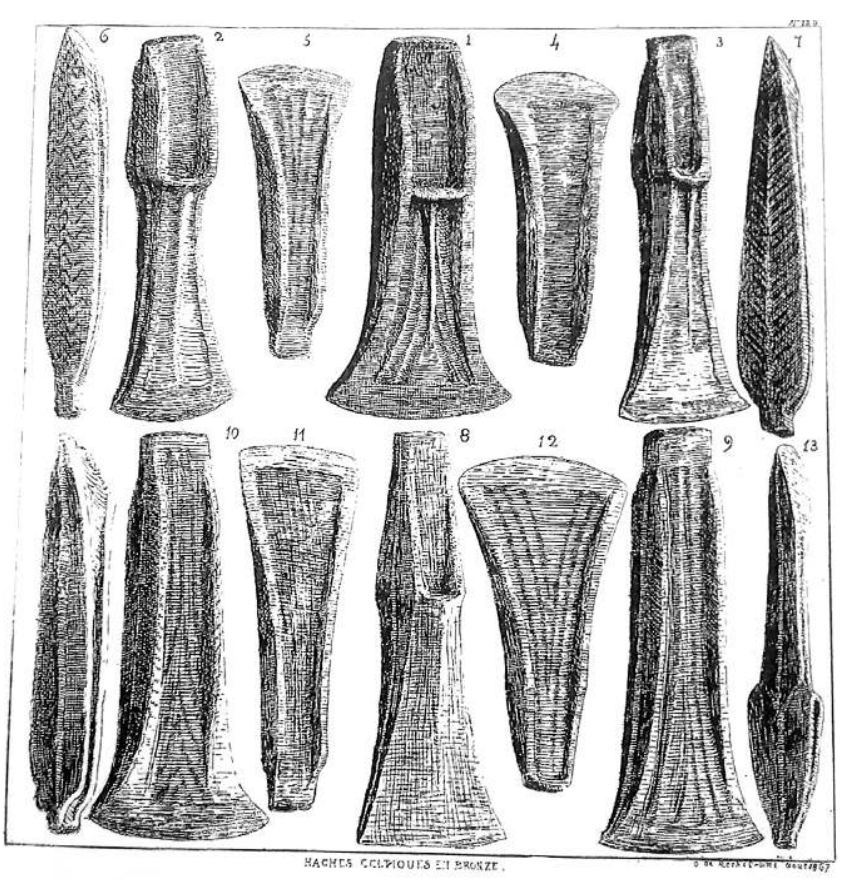

Figure 4 : Première figuration d'une sélection de haches du dépôt de Petosse. Planche gravée datée de 1867 (Rochebrune, 1880).

Figure 4: First drawings dating from 1867 of a selection of axes from the Petosse hoard (Rochebrune, 1880).

Plus loin, il signale que la plupart sont du type à rebords : " sur soixante types, il y en a au moins cinquante de cette espèce » [note de bas de page p. 14]. Les autres sont de type à talon, et sont au nombre de six.

L'acquisition des objets s'est faite assez rapidement après la découverte, comme le précise lui-même O. de Rochebrune dans son article de 1901 sur Massigny, et dans lequel il dit s'être procuré les objets plus de trente ans auparavant. La planche des haches de Petosse apporte plus de précisions, puisqu'elle est datée d'août 1867 (fig. 4).

\section{Concernant le nombre de haches déposées}

Le nombre exact de haches comprises dans le dépôt de Petosse reste assez obscur : O. de Rochebrune (1880) parle d'abord de "soixante et quelques haches ", p. 11, puis de "soixante haches ", p. 12, et encore de "soixante types", p. 14.

Le nombre généralement suivi est celui donné par l'abbé Breuil, d'après son propre comptage lors de l'étude des haches alors passées dans les collections de Raoul de Rochebrune, à savoir : 55 haches, un chiffre assez différent donc de celui donné par O. de Rochebrune. Parmi ces 55 haches, il décompte 47 haches à rebords et 8 à talon. Lorsqu'il mène son étude, il précise que 3 haches font partie

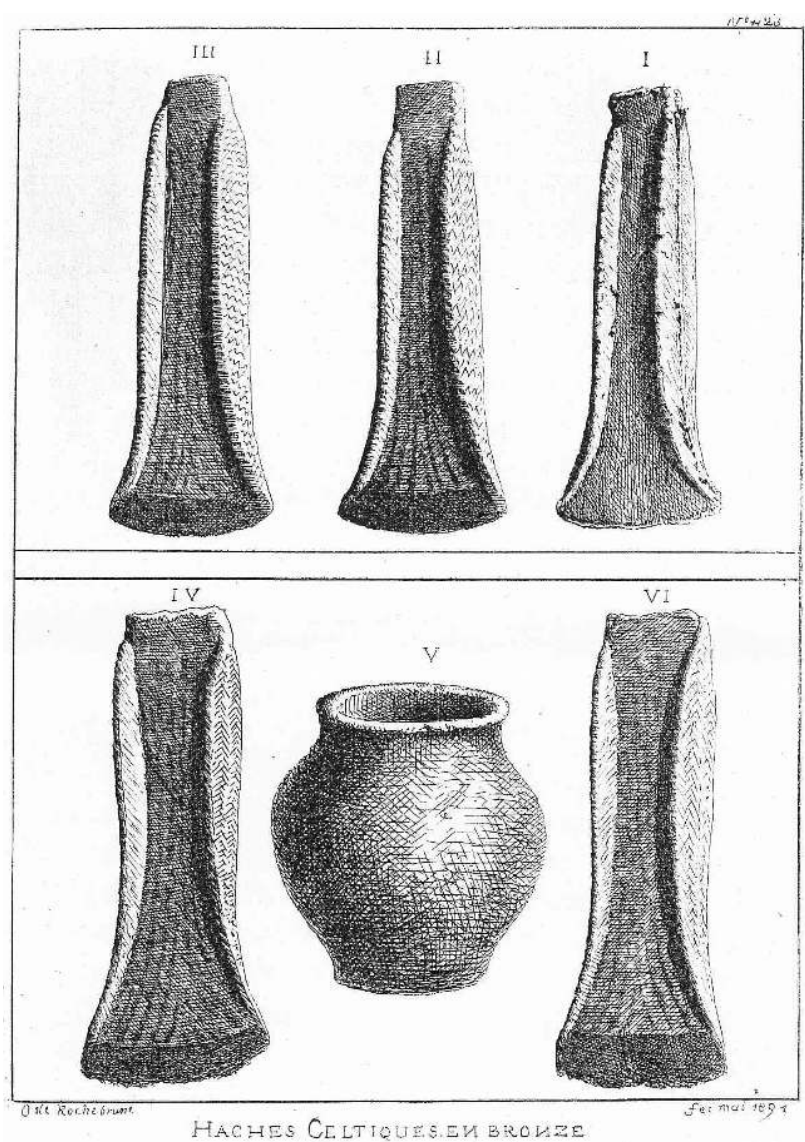

Figure 5 : Planche figurant deux des haches du dépôt de Chaix, La Roche Courion ("Massigny ") ainsi que la poterie dans laquelle elles étaient renfermées (en bas) et trois haches de Petosse (en haut) (Rochebrune, 1901).

Figure 5: Bottom: two axes from the Chaix hoard with their pottery vessel; top: three axes from the Petosse hoard (Rochebrune, 1901).

d'autres collections : 2 haches sont en possession de G. de Fontaines $^{3}$ et 1 en possession de G. Chauvet, à Ruffec.

Plus de 60 ou 55 haches? O. de Rochebrune donne un indice intéressant pour régler cette question : la totalité du dépôt pèse environ 60 livres, soit environ $30 \mathrm{~kg}$ (Rochebrune, 1880 , p. 12). Or, si on fait le calcul à partir d'une part de la masse moyenne des haches à rebords ${ }^{4}(478 \mathrm{~g}$ pour 1 individu) et d'autre part de la masse totale des haches à talon nouvellement identifiées (2415 g; voir infra), en y ajoutant la hache à rebords et à talon $(623 \mathrm{~g})$, le nombre donné par Breuil donnerait une masse totale d'environ $27 \mathrm{~kg}$, ce qui semble faible par rapport à celle donnée par O. de Rochebrune. Si l'on effectue ce calcul sur 60 haches,

3. Elles étaient jusqu'à il y a peu en possession de ses descendants, et sont entrées récemment dans les collections de l'Historial de la Vendée, aux Lucs-sur-Boulogne.

4. On se base ici sur la masse moyenne établie sur 90 individus à rebords de type vendéen (Mélin, 2014). 
la masse totale serait d'environ 28372 g, et de $29806 \mathrm{~g}$ si l'on fait ce calcul sur 63 haches. Il semble par conséquent plus probable que le nombre total de haches contenues dans le dépôt de Petosse ait été plus proche de 60 comme dit par O. de Rochebrune (62? 63 ?), que de 55 comme écrit par l'abbé Breuil.

Étant donné que Breuil ne reprend pas un nombre donné par un tiers mais bien un comptage effectué par lui-même, on peut se demander si, à l'instar des trois haches vraisemblablement données par $\mathrm{O}$. de Rochebrune après son acquisition du dépôt à $G$. Chauvet et $G$. de Fontaines, d'autres haches n'auraient pas fait l'objet de dons ou d'échanges entre 1880 et 1902. Il semblerait en effet que quelque sept haches pouvaient manquer au moment de l'étude de l'abbé Breuil. Si tel est le cas, celles-ci pourraient être repérables encore aujourd'hui par le biais de la patine très caractéristique que présentent les haches de Petosse.

\section{Localisation actuelle des haches}

À l'occasion de l'étude du dépôt de Mouilleron-en-Pareds, des recherches dans les collections du musée départemental Dobrée nous ont amenée à redécouvrir plusieurs haches provenant de ces deux dépôts. Si quelques-unes des haches avaient été mises en parallèle avec les haches décorées publiées par l'abbé Breuil par les agents du musée, il nous semble possible d'en adjoindre plusieurs autres sans provenance indiquée et ce, grâce à une patine similaire. La patine des objets en alliage cuivreux est souvent problématique car très variable. Ici elle permet au contraire de regrouper un grand nombre d'objets issus du même ensemble à l'origine à partir d'un groupe de base de provenance certaine, et ce avec beaucoup de probabilité. En effet, la patine des haches de l'ensemble de Petosse s'avère très reconnaissable : leur état de conservation est tout d'abord très bon. La patine est d'un vert mat homogène et un reste de sédiment orangé apparaît sur chacune. Ceci est cohérent avec la description de O. de Rochebrune : il fait état en effet d'une argile, une " terre rouge " qui n'avait pas été enlevée par le découvreur, mais retirée " avec soin " par lui-même (Rochebrune, 1880 , p. 12). Nous avons donc rassemblé plusieurs haches présentant cette patine et ces restes de sédiments. Un élément important venant confirmer l'intérêt de s'appuyer sur la patine pour cet ensemble et valider les regroupements effectués est l'existence de deux haches ayant été conservées dans des conditions différentes de celles retrouvées dans les réserves du musée, pendant plus d'un siècle : ces deux haches, qui ont très tôt appartenu à la collection G. de Fontaines (précisé par Breuil, 1902a), montrent en effet une patine absolument similaire à celles conservées au musée Dobrée.

\section{Des Confusions ENTRE LeS DÉPÔTS de Petosse et Chaix (« Massigny »)}

\section{Les premières figurations par $\mathrm{O}$. de Rochebrune}

Les haches du dépôt de Petosse apparaissent pour la première fois sur une planche signée $\mathrm{O}$. de Rochebrune et publiée dans sa monographie présentant sa collection d'armes (Rochebrune, 1880). Il est intéressant de noter la date de la réalisation de ladite planche, visible près de la signature : " août 1867 " (fig. 4). Cette planche sera republiée à l'identique dans l'ouvrage de B. Fillon et O. de Rochebrune, Poitou et Vendée. Études historiques et artistiques en 1887, mais, bien qu'elle ait été insérée dans la publication, aucune référence n'y est faite.

Deux haches du dépôt de Chaix apparaissent dans l'article d'O. de Rochebrune en 1901 dans la Revue du Bas-Poitou, article dans lequel, outre la présentation de la découverte du torque en or de Velluire, il mentionne la découverte faite dix ans plus tôt à la Roche Courion à Chaix (fig. 5). En haut sont figurées trois haches du dépôt de Petosse; en bas, deux des trois haches de Chaix, encadrant une restitution de la poterie dans laquelle elles étaient placées. Cette disposition permet à $\mathrm{O}$. de Rochebrune de faire des parallèles entre ces deux dépôts contenant des haches "fondues dans les mêmes moules et martelées de la même façon ", mais aussi d'indiquer des différences intéressantes au niveau de l'état du fil du tranchant, puisque les haches de la Roche Courion montrent des ébréchures, bien représentées sur ses dessins, alors que celles de Petosse possèdent des tranchants intacts, "sans écornures ", qui donc " n'avaient jamais servi ». Ce détail sur l'état des tranchants sera important pour la reconnaissance des haches de Chaix.

\section{Les haches de « Massigny » selon l'abbé Breuil}

Très rapidement sortent deux articles publiés par l'abbé Breuil, en 1902. La première publication (Breuil, 1902a) dans la Revue archéologique donne une provenance erronée des haches illustrées (Saint-Étienne-de-Brillouet), mais elle est rapidement corrigée dans le deuxième article (Breuil, 1902b). Quinze haches sont figurées, trois de " Massigny " - c'est-àdire la Roche Courion à Chaix - et douze de Petosse; il s'agit de haches à talon et de haches à rebords de type vendéen.

Celles indiquées par Breuil comme provenant de ChaixMassigny sont les numéros 8, 9 et 11 de son article (1902b; ici fig. 6).

Si l'on compare ces trois haches avec les dessins des deux haches figurées par O. de Rochebrune en 1901 (fig. 5), on peut estimer que la $\mathrm{n}^{\circ} 9$ de Breuil correspond effectivement à la hache $n^{\circ} \mathrm{IV}$ d'O. de Rochebrune (même décor en dents 
de loups, même petite lacune d'un angle du tranchant semble-t-il). Par contre, la deuxième hache figurée par O. de Rochebrune ne ressemble pas vraiment aux deux indiquées comme provenant du même dépôt par l'abbé Breuil : la no 8 présente un décor différent sur le profil et un tranchant bien plus convexe; elle est également plus petite; la $\mathrm{n}^{\circ} 11$ semble déjà plus proche à quelques détails près, mais nous verrons plus loin que cette dernière correspond en tout point à une autre hache (fig. 7).

Figure 6 : Les trois haches du dépôt de Chaix-Massigny selon H. Breuil (1902b).

Figure 6: The three axes from the Chaix hoard of ("Massigny") according to H. Breuil (1902b).

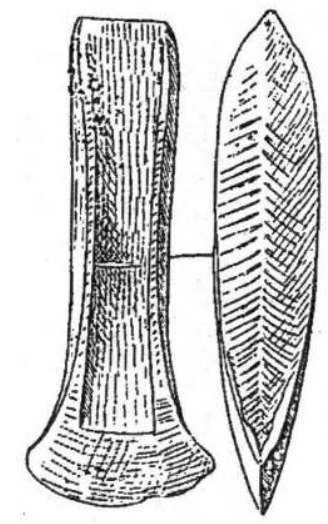

\section{Ré-identification des haches de Chaix, la Roche Courion («Massigny»)}

D'après nos recherches parmi les haches issues des collections de Rochebrune léguées au musée Dobrée, une hache parait bien correspondre à la hache ${ }^{\circ} \mathrm{IV}$ d'O. de Rochebrune et $\mathrm{n}^{\circ} 9$ de l'abbé Breuil : il s'agit de celle portant le numéro d'inventaire 930.1.224 (fig. 8). On retrouve la même forme du tranchant qui remonte brusquement au niveau d'une des

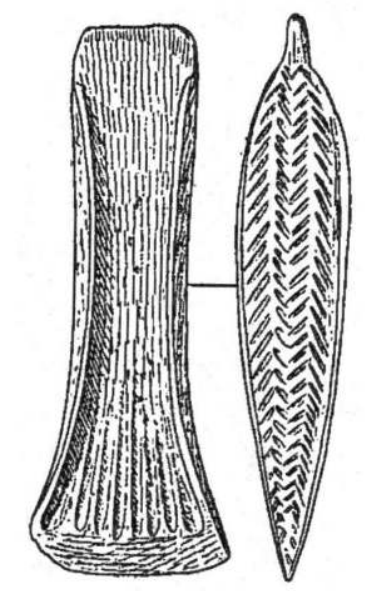

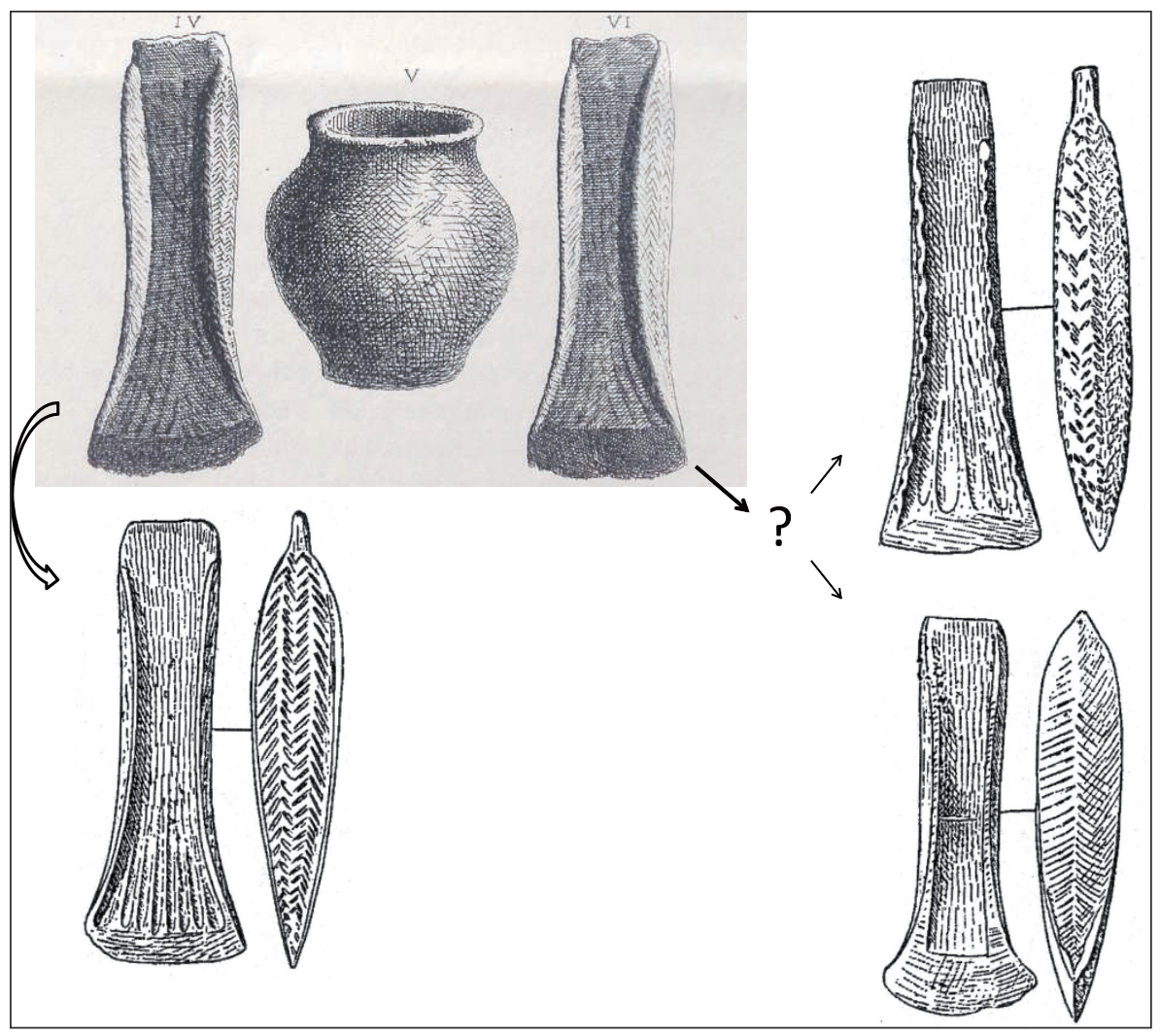

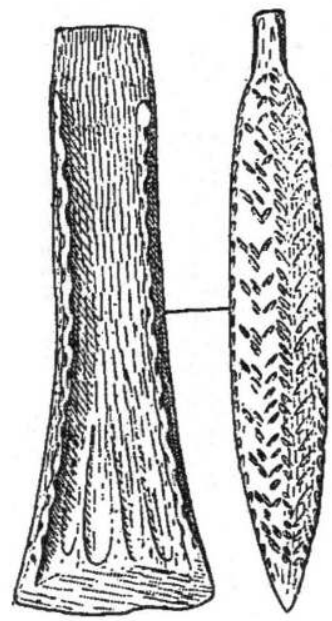

Figure 7 : Recherche de correspondances entre la planche d'O. de Rochebrune (1901) figurant deux des haches de Chaix, la Roche Courion (ou " Massigny ") et les dessins donnés par H. Breuil (1902b) : la hache $\mathrm{n}^{\circ} 9$ de $\mathrm{H}$. Breuil semble correspondre à la hache $\mathrm{n}^{\circ}$ IV d'O. de Rochebrune. Par contre, rien n'est moins sûr pour les deux autres haches attribuées à " Massigny " par H. Breuil (voir fig. 11 et 12). Figure 7: Comparison of the Chaix axes illustrated in 1901 by $O$. de Rochebrune and those illustrated by H. Brevil in 1902: the axe $n^{\circ} 9$ in Breuil's drawing seems to correspond to the $n^{\circ} I V$ of $O$. de Rochebrune's drawing. The correspondence of the two other axes remains unclear (cf. fig. 11 and 12). 
cornes latérales, les cannelures sur les plats et un décor similaire sur le profil. Il faut ici souligner la patine particulière de cet objet, brillante, brune et dorée, en particulier sur les profils, avec des taches vertes par endroits. Il semble que ces haches aient été " nettoyées " après leur découverte

Cette patine nous a mis sur la piste de la deuxième hache figurée dans l'article de Rochebrune, la ${ }^{\circ}$ VI, qui semble bien être la hache portant le numéro d'inventaire 930.1.221 (fig. 9). On retrouve en effet, outre un décor similaire de cannelures sur les plats et en dents de loups sur les profils, des détails d'ébréchures similaires au niveau du tranchant. La patine est par ailleurs analogue, bien que plus verte sur le plat présenté en photographie, mais avec le même aspect "nettoyé ", en particulier sur le profil qui montre un aspect très doré.

Reste enfin une dernière hache provenant du dépôt de Chaix malheureusement non figurée par O. de Rochebrune. Une hache sans provenance des collections du musée Dobrée, portant le numéro 56.2306, nous semble pouvoir tout à fait remplir ce rôle : il s'agit d'une hache de type vendéen, à cannelures sur le plat et à décor martelé horizontalement sur les profils (fig. 10). Elle présente une patine dorée et sombre, avec taches vertes. Son aspect "nettoyé " faisant ressortir la couleur du bronze ressemble clairement à celle des haches précédemment décrites, et bien que cette troisième et dernière hache ne soit pas figurée par $\mathrm{O}$. de Rochebrune, il nous semble très probable qu'il s'agisse de celle-ci, aucune autre hache des collections du musée ne présentant de similitudes avec les haches de Chaix.

Parallèlement, et pour écarter définitivement la hache $\mathrm{n}^{\circ} 11$ illustrée par l'abbé Breuil dans son article comme provenant du dépôt de Chaix, la Roche Courion, nous pouvons nous appuyer sur une hache à rebords appartenant à la collection de Rochebrune, enregistrée sous le numéro 930.1.225, et ressemblant en tout point à celle dessinée par Breuil, du décor très particulier sur le profil et celui des rebords, jusqu'au détail d'une petite cassure, ancienne, au niveau du tranchant (fig. 11). On remarquera également la forme du profil, beaucoup plus rectiligne que pour la forme classique en foliole de feuille de marronnier, avec un sommet assez haut et épais, caractéristiques qui correspondent à un soustype 2 que nous avons défini pour le type vendéen (Mélin, 2014). Pour finir, cette hache présente sans aucune ambiguiité la patine très caractéristique des haches du dépôt de

5. Si tel est le cas, ce n'est sans doute pas du fait d'O. de Rochebrune, mais celui du découvreur lui-même si l'on en croit la remarque du collectionneur, en 1880 pour les haches de Petosse : «C'est une grande joie pour l'antiquaire lorsque l'objet découvert n'a pas été nettoyé [...] 》 (note de bas de page (I), p. 12). Outre une patine "nettoyée », cette hache présente des stries modernes à différents endroits. Le tranchant lui-même semble montrer des stigmates récents.

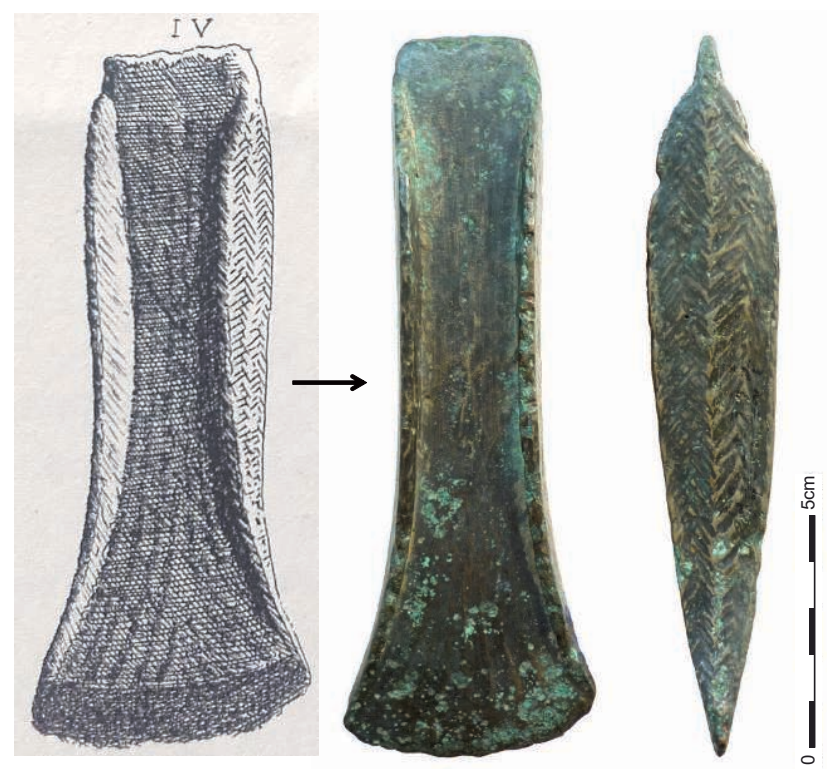

Figure 8 : Une des trois haches du dépôt de la Roche Courion à Chaix ("Massigny ») conservée au musée archéologique de Nantes ( ${ }^{\circ}$ 930.1.224) correspond au dessin $n^{\circ}$ IV d'O. de Rochebrune, 1901 (dessin O. de Rochebrune; cliché M. Mélin).

Figure 8: One of the three axes from the Chaix hoard (Rochebrune, $1901 n^{\circ} I V$ ) corresponds to an axe preserved in the collection of the archaeological museum of Nantes ( $n^{\circ}$ 930.1.224; photo M. Mélin).

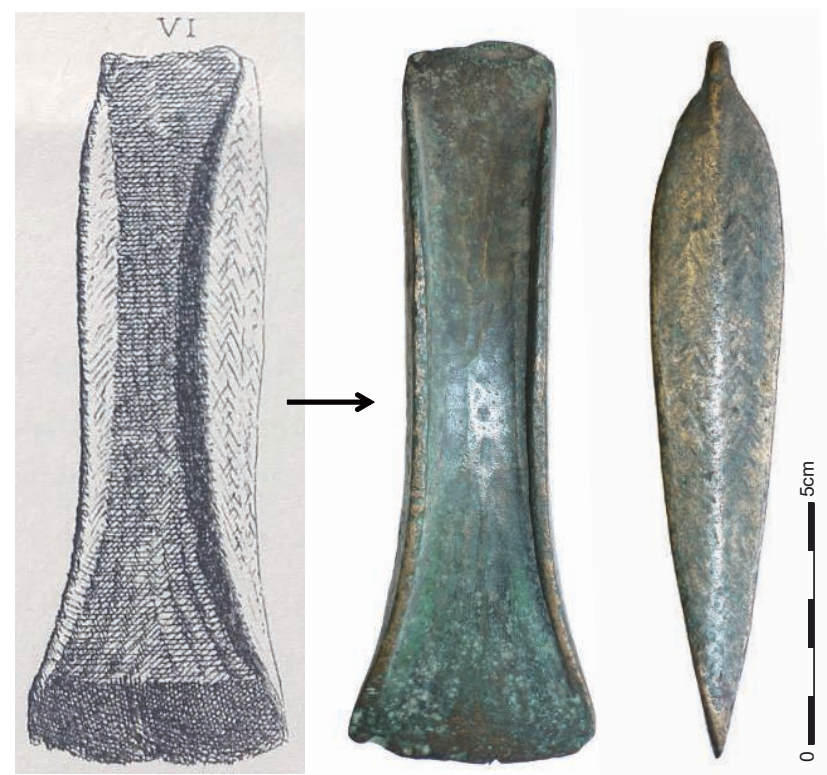

Figure 9 : Une autre hache du dépôt de Chaix, la Roche Courion ("Massigny ") retrouvée au musée archéologique de Nantes ( ${ }^{\circ}$ 930.1.221) correspond au dessin $n^{\circ}$ VI d'O. de Rochebrune, 1901 (dessin O. de Rochebrune; cliché M. Mélin).

Figure 9: Another axe from the Chaix hoard (Rochebrune, 1901 $\left.n^{\circ} V I\right)$ corresponds to an axe preserved in the collection of the archaeological museum of Nantes ( $n^{\circ}$ 930.1.221 - photo M. Mélin). 


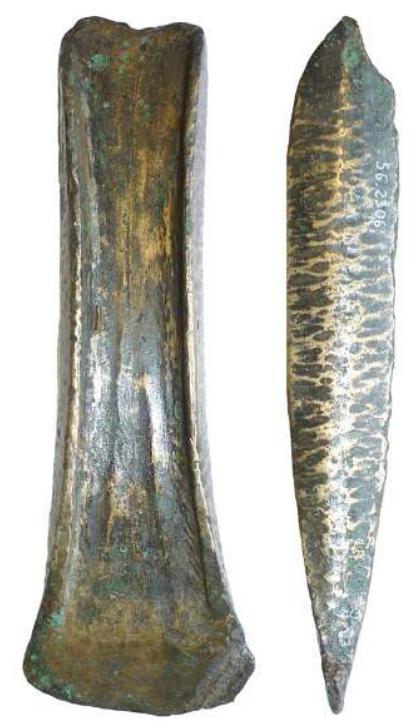

Figure 10 : Hache du musée archéologique de Nantes montrant une patine " nettoyée " tout à fait similaire aux deux haches précédentes. Malgré l'absence d'illustration de la troisième hache du dépôt de Chaix, la Roche Courion par O. de Rochebrune, il y a de très fortes probabilités pour qu'il s'agisse de celle-ci, $n^{\circ} 56.2306$ (cliché M. Mélin).

Figure 10: Flanged-axe preserved in the museum of Nantes, showing a "leaned-up" patina very similar to the previous two axes (fig. 8 and 9). Despite the absence of an illustration of the third axe of Chaix, it is highly probable that this is the axe in question (photo M. Mélin).
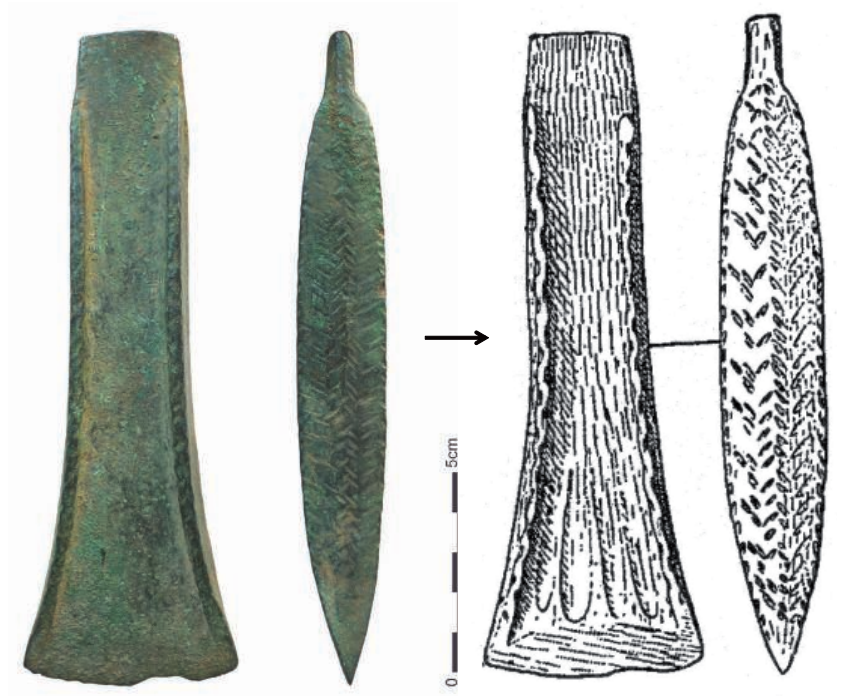

Figure 11 : Correspondance entre la hache 930.1.225 du musée et le dessin $n^{\circ} 11$ de $\mathrm{H}$. Breuil. Alors que H. Breuil la fait provenir de "Massigny " (c'est-à-dire la Roche Courion à Chaix), cette hache possède la patine caractéristique des haches de Petosse (cliché M. Mélin; dessin H. Breuil).

Figure 11: Comparison between the axe $n^{\circ} 11$ of H. Breuil (1902b) and the axe $n^{\circ}$ 930.1.225 of the museum of Nantes. Attributed to "Massigny" by H. Breuil, it shows without doubt the very characteristic patina of the axes of Petosse (photo M. Mélin).
Petosse. Il est d'ailleurs possible que ce soit ce profil qui apparaisse sur la planche d'O. de Rochebrune, publiée en 1880, sous le numéro 6 (fig. 4). Dans tous les cas, elle permet de confirmer que les attributions de l'abbé Breuil en 1902 sont erronées et qu'il y a eu des confusions et mélanges entre les deux dépôts.

La petite hache ${ }^{\circ} 8$ figurée par H. Breuil en 1902 et qu'il donne comme venant du dépôt dit de Massigny trouve, elle, un écho très convaincant dans une des haches conservées au musée Dobrée sous le numéro d'inventaire 930.1.222 : il s'agit d'une hache à rebords de taille un peu réduite avec un décor en arête de poisson sur le profil (fig. 12). On note la même forte convexité du tranchant que pour la hache dessinée par l'abbé Breuil, tranchant possédant la même petite cassure, et les mêmes rebords nettement aplatis au-dessus du tranchant. Il s'agit bien du même objet. Nous reviendrons plus loin sur une petite particularité que présente cet objet, longtemps considéré comme un critère typochronologique d'après le dessin qu'en avait donné l'abbé Breuil. Cette hache présente très clairement la patine des haches de Petosse, vert mat avec les restes du même sédiment.

Nous avons donc pu démontrer les erreurs d'attribution données par Breuil dans son deuxième article de $1902^{6}$, et les mélanges faits avec les haches de Petosse, deux dépôts possédés par $\mathrm{O}$. de Rochebrune, ceci grâce au retour sur ces objets, et grâce à leur patine très caractéristique.

Pour résumer, les correspondances pour les trois haches du dépôt de Chaix, la Roche Courion, entre leurs numéros d'inventaire actuels et les dessins figurés dans Breuil, 1902b et de Rochebrune, 1901, apparaissent dans le tableau 1. Les haches du dépôt de la Roche Courion à Chaix, anciennement dit de Massigny, sont donc ré-identifiées et leur lieu de conservation désormais connu.

\section{Ré-identification des haches de Petosse}

La collection de Rochebrune fait partie du musée archéologique départemental de Nantes depuis 1930. Tout comme pour le dépôt de Chaix, la Roche Courion, il semble que les haches du dépôt de Petosse se soient retrouvées dans les collections du musée tout en restant inconnues des chercheurs ayant travaillé sur le sujet (Pautreau, 1979; Gomez de Soto, 1995; Poissonnier, 1997). Plusieurs haches du musée correspondant aux dessins de l'abbé Breuil ont bien été identifiées

6. L'abbé Breuil avait bien noté des différences de patine entre les haches : "Toutefois, j'avais remarqué que plusieurs haches ornées (3) n'avaient aucune patine, mais j'avais attribué cela à un nettoyage destiné à faire ressortir les ornementations. En réalité, il s'agissait d'une seconde trouvaille. " (1902b). Malgré cela, il ne réattribue pas aux bonnes haches la bonne provenance. Peut-être n'a-t-il pas pu retourner sur les objets avant les modifications de son deuxième article. 


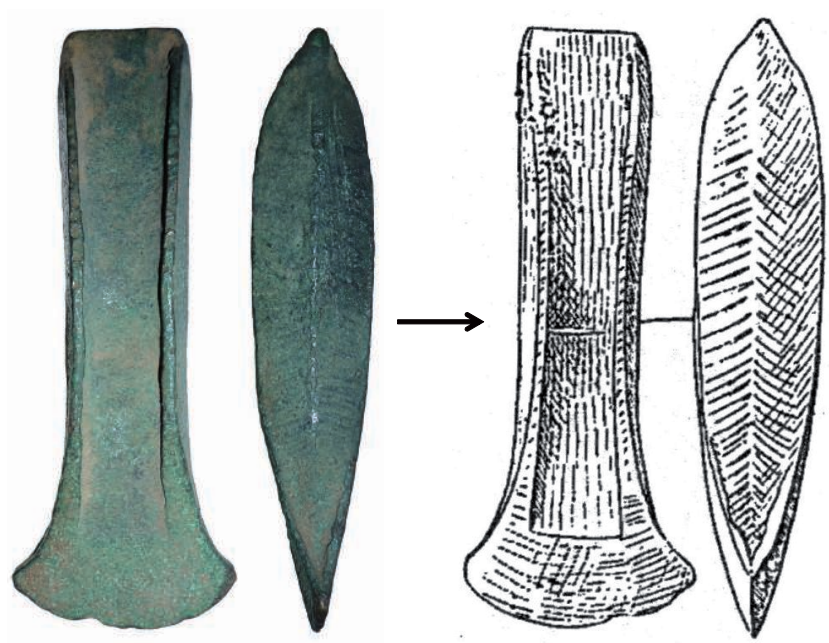

Figure 12 : Correspondance entre la hache $\mathrm{n}^{\circ} 8$ de H. Breuil (1902b) et la hache $n^{\circ}$ 930.1.222 du musée archéologique de Nantes. Bien qu'attribuée au dépôt de " Massigny » par H. Breuil, la patine de cette hache montre sans aucun doute la patine caractéristique des haches de Petosse (cliché M. Mélin; dessin H. Breuil). Figure 12: Comparison between the axe $n^{\circ} 8$ of H. Breuil (1902b) and the axe $n^{\circ}$ 930.1.222 of the museum of Nantes. Attributed to "Massigny" by H. Breuil, it shows without doubt the very characteristic patina of the axes of Petosse (photo M. Mélin).

\begin{tabular}{|c|c|c|c|}
\hline Objet & $\begin{array}{l}\mathrm{n}^{\circ} \text { inventaire } \\
\text { musée Dobrée }\end{array}$ & $\begin{array}{c}\text { dessin } \\
\text { dans } \\
\text { Breuil, } \\
\text { 1902b }\end{array}$ & $\begin{array}{c}\text { dessin dans } \\
\text { Rochebrune, } \\
1901\end{array}$ \\
\hline $\begin{array}{l}\text { Hache à rebords, } \\
\text { de type vendéen }\end{array}$ & 930.1 .224 & fig. 9 & $\mathrm{n}^{\circ} \mathrm{IV}$ \\
\hline $\begin{array}{l}\text { Hache à rebords, } \\
\text { de type vendéen }\end{array}$ & 930.1 .221 & non & $\mathrm{n}^{\circ} \mathrm{VI}$ \\
\hline $\begin{array}{l}\text { Hache à rebords, } \\
\text { de type vendéen }\end{array}$ & 56.2306 & non & non \\
\hline
\end{tabular}

Tableau 1 : Haches contenues dans le dépôt de la Roche Courion à Chaix (aussi dit de "Massigny ") après corrections des attributions erronées de l'article de H. Breuil (1902b), et correspondances établies entre les dessins de son article et celui d'O. de Rochebrune (1901) et d'après patine.

Table 1: Axes of the la Roche Courion hoard in Chaix ("Massigny") after the correction of wrong attributions made by $H$. Breuil, and comparison between his drawings and the ones of O. de Rochebrune (1901).

comme provenant de Petosse, celles qui sont décorées principalement. Un grand nombre d'autres portant une patine très reconnaissable sont également présentes au musée, mais sans attribution de provenance. Nous avons en particulier retrouvé une des haches de Petosse assez originale pour être facilement reconnaissable : il s'agit de la hache à rebords et butée à décor de légères cannelures sur la lame figurée par
H. Breuil au no 13 (ici fig. 13). Cette hache (n 56.2313 ) et une hache de type Centre-Ouest ( ${ }^{\circ} 56.2311$; ici, fig. 14) ont été restaurées pour leur présentation dans la dernière exposition permanente du musée. Par ce nettoyage, elles portent désormais une patine différente, d'un vert plus sombre, plus brillant que celles des autres haches, et ne conservent plus de sédiment. Heureusement, leur provenance est assurée par leur correspondance avec les figurations de l'abbé Breuil. On notera par cet exemple la perte d'information que peuvent potentiellement entrainer les restaurations ou le nettoyage des objets ayant perdu leur provenance.

La patine des haches de Petosse est si reconnaissable que nous avons pu rassembler un groupe de haches dont l'appartenance originelle à cet ensemble ne nous semble pas faire de doute. C'est le cas de plusieurs haches à talon sans provenance connue qui correspondent aux dessins de Breuil (fig. 14). Au total, nous avons regroupé cinq haches à talon de type breton et de type Centre-Ouest des collections du musée Dobrée, auxquelles s'ajoute une hache de type Centre-Ouest conservée jusqu'à il y a peu dans la collection de Fontaines. Combien y en avait-il à l'origine? Breuil parle de huit haches à talon alors que Rochebrune n'en mentionne que six. On ne sait si l'un et l'autre comptabilisent la hache à rebords et à talon. Sans savoir si elles sont au complet, nous en comptabilisons six, plus celle à rebords et à talon.

Il est intéressant de s'arrêter sur le fait qu'O. de Rochebrune précise qu'il y a une variabilité au niveau de la taille des haches de Petosse (Rochebrune, 1880, p. 14) : cer-
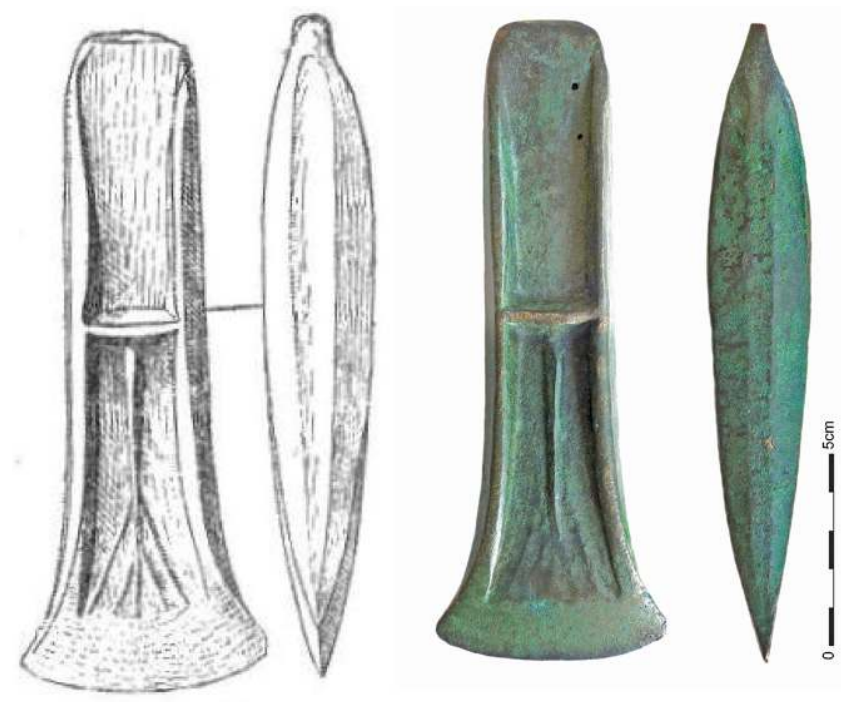

Figure 13 : Hache à rebords et à talon du dépôt de Petosse (dessin H. Breuil [1902] et photographie de la hache $\mathrm{n}^{\circ} 56.2313$, M. Mélin).

Figure 13: Flanged-axe with a stop ridge from the Petosse hoard that was labelled "unprovenanced" in the collection of the museum of Nantes - n 56.2313 (drawing H. Breuil, 1902; photo M. Mélin). 


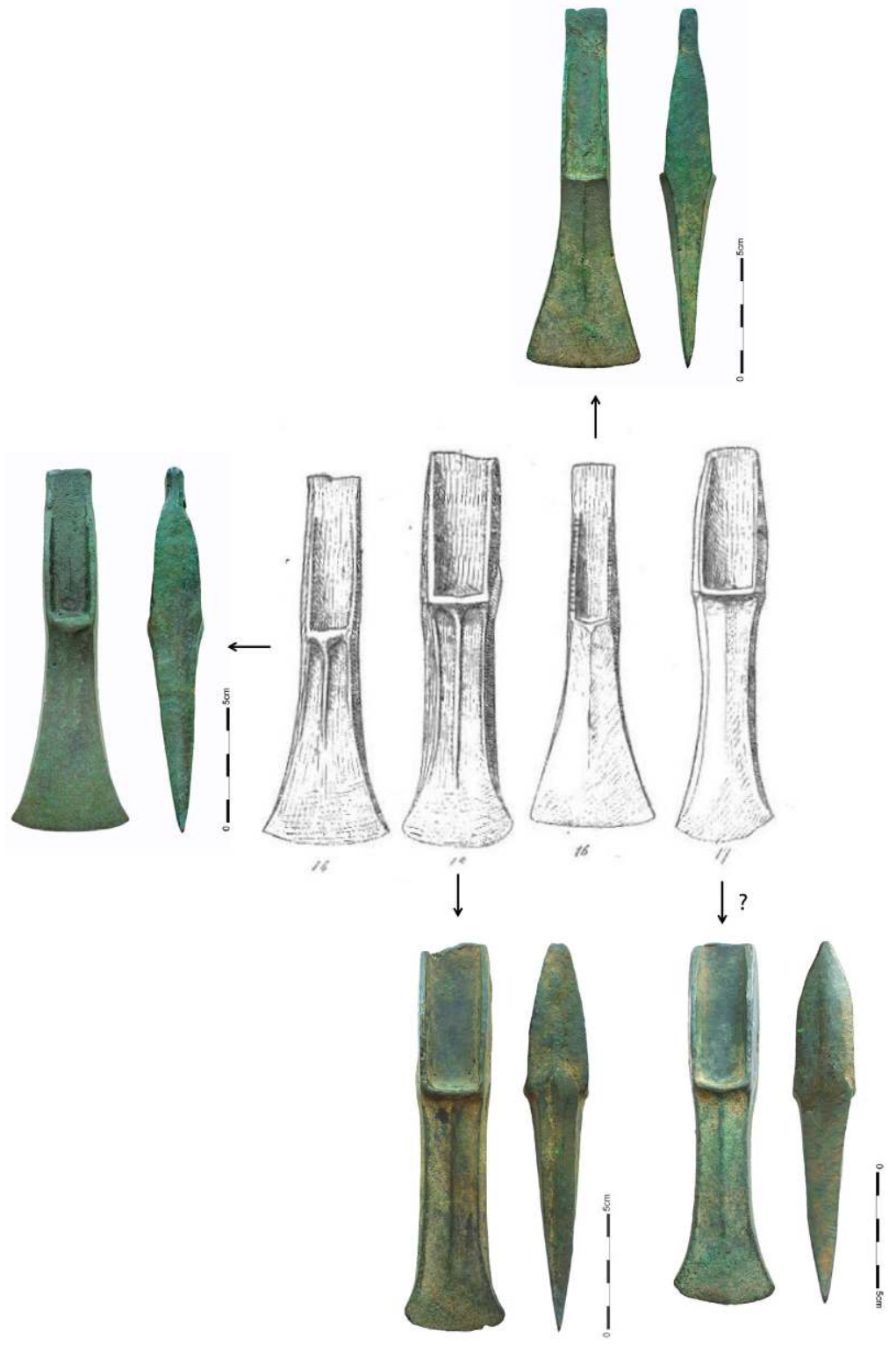

Figure 14 : Correspondances entre les haches figurées par $\mathrm{H}$. Breuil et plusieurs haches à patines similaires (sauf une, restaurée) conservées au musée Dobrée (dessin H. Breuil; cliché M. Mélin).

Figure 14: Comparison between the axes illustrated by $H$. Breuil and several axes with a similar patina (one restored axe exempted) from the collections of the museum of Nantes (drawing H. Breuil, 1902; photo M. Mélin). taines sont " petites, courtes, minces, légères " $\left(\mathrm{n}^{\text {os }} 4\right.$ et 5 de sa planche, ici fig. 4), alors que d'autres sont plus grandes et plus lourdes. Cette variabilité se retrouve au sein des haches que nous avons regroupées. En effet, trois d'entre elles, que nous proposons d'attribuer au dépôt de Petosse peuvent être qualifiées de miniatures. Elles doivent correspondre aux « deux ou trois » haches que l'abbé Breuil met en exergue par cette particularité : elles sont, selon lui ${ }^{7}$, « raccourcies par un long usage " (1902b, p. 929). O. de Rochebrune mentionne par ailleurs une hache au tranchant en éventail : la n ${ }^{\circ} 12$ de sa planche (ici fig. 4). Cette hache fait plus penser au type libournais (Lagarde-Cardona, 2012) qu'au type vendéen. Il

7. Mais cela ne nous semble pas être le cas. y a bien une hache de type libournais sans provenance dans les collections du musée Dobrée, mais celle-ci possède une patine noire très différente.

Parmi les haches de Petosse, certaines sont décorées, d'autres sont " lisses ", nous dit O. de Rochebrune (1880, p. 14) : c'est le cas des haches que nous avons regroupées. Il précise également qu'une seule d'entre elles est " brute de fonte " (ibid., p. 13) : une seule des haches rassemblées ( $\mathrm{n}^{\circ}$ 56.2293), sans être strictement brute de fonte, se distingue bien des autres par le fait qu'elle a été très peu reprise après fonte (elle n'a subi qu'un premier ébarbage sommaire). La présence d'un unique cas de hache à cet état d'ébauche au sein du dépôt rappelle curieusement le dépôt contemporain de Mouilleron-en-Pareds (Vendée) qui 
a également livré une hache dans cet état pour 51 au total (Mélin, 2011 et 2012).

Par contre, nous ne voyons pas à quoi se rapporte cet autre détail que donne O. de Rochebrune : « une seule porte des traces encore très visibles d'étamure " (Rochebrune, 1880).

S'ajoutent à cela les deux haches restées dans la collection privée de G. de Fontaines mentionnées par Breuil en 1902 - l'une d'elles a d'ailleurs été dessinée par ce dernier : la $\mathrm{n}^{\circ} 10$ - et plus récemment republiées (Le Quellec, 1989). Nous avions également eu l'occasion d'étudier ces deux haches en 2011. Elles ont été déposées depuis peu à l'Historial de la Vendée 8 . Nous avons déjà mentionné l'intérêt de retrouver ces haches et de les comparer aux haches du musée Dobrée : leur patine est strictement comparable, et ce malgré un siècle de conservation dans deux lieux et donc dans des conditions différentes, ce qui valide cet exercice de réattribution de haches sans provenance via leur patine notamment. Une autre hache, "à bords droits sans ornementation » (Rochebrune, 1880), a rapidement été donnée à G. Chauvet, archéologue de Ruffec (Breuil, 1902a). Son lieu de conservation n'est toujours pas connu à l'heure actuelle (musée Sainte-Croix de Poitiers?).

Au total et à ce jour, seules 39 haches semblent pouvoir être réattribuées au dépôt de Petosse (tabl. 2). Ce chiffre est encore trop faible par rapport au nombre de haches constituant le dépôt à l'origine. Une vingtaine d'objets reste encore introuvable et les recherches sont donc à poursuivre. L'espoir de retrouver ces haches est grand vu la qualité de la patine des objets, très reconnaissable.

Pour finir sur le dépôt de Petosse, arrêtons-nous sur la hache ${ }^{\circ}$ 930.1.222, présentée sur la figure 12. Le dessin de l'abbé Breuil, qui la fait provenir de Massigny, a pu induire en erreur et inciter à donner une datation haute à ce dépôt, et par extension au type vendéen : sur son dessin en effet, la hache semble présenter une déclivité du plat, environ à mihauteur de l'objet (Breuil, 1902b; Pautreau, 1979, p. 140, 147) : un trait figuré et des ombrages indiqueraient ainsi une légère butée comme l'on en connait sur les haches dites à butée médiane (Blanchet, Mordant, 1987). Or, il ne s'agit pas là d'une butée, mais d'un creux, correspondant à un défaut de coulée (fig. 15). Cette confusion typologique a eu une incidence importante puisque jouant sur la position chronologique des haches de type vendéen. Cet objet ne peut donc être pris en exemple pour discuter d'une datation haute du type vendéen. Par contre, la hache no 56.2313 (fig. 13) du même dépôt possède bien un aspect archaïque, par la présence de rebords conjointement à une butée ayant la même hauteur, qualifiable de talon.

8. Information Sophie Corson (Historial de la Vendée).

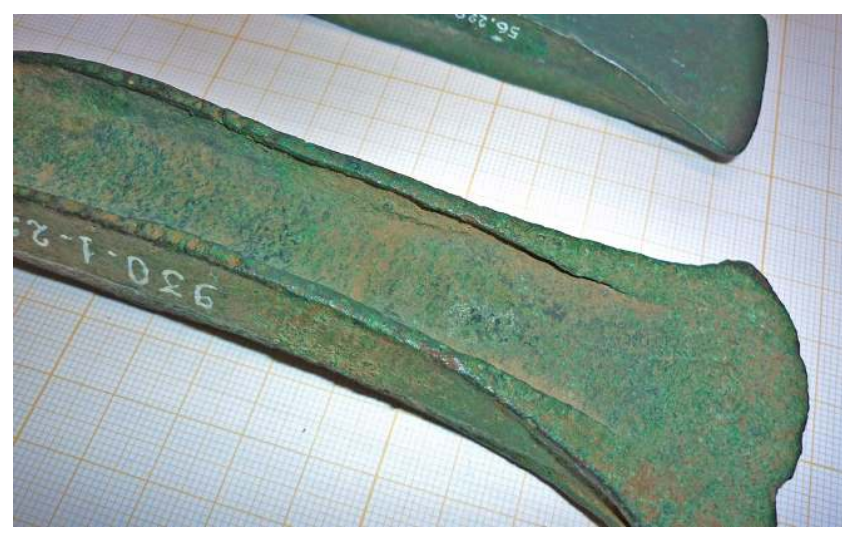

Figure 15 : Contrairement à ce qu'a pu laisser penser le dessin de H. Breuil (1902b, no 8), il n’y a pas de butée médiane sur le plat de cette hache (cliché M. Mélin).

Figure 15: Contrary to what could be interpreted from the drawing of $H$. Breuil (1902b, no 8), there is no stop ridge on this axe (photo M. Mélin).

\section{Conclusion}

Cette contribution aura permis de faire un peu de " ménage " dans deux importants dépôts vendéens, et de reparler de l'ensemble de Petosse, qui, avec ceux de Mouilleron-en-Pareds (Mélin, 2012; 2014) et de SaintMathurin', est un des plus gros dépôts du département pour le Bronze moyen 2. Ces trois dépôts sont sur plusieurs points tout à fait comparables, outre leur importance numérique (Mélin, 2015). Si les haches du dépôt de Saint-Mathurin sont malheureusement dans un état de conservation médiocre, ce n'est pas le cas de celles des deux autres dépôts.

Le dépôt de Mouilleron-en-Pareds a fait l'objet d'une étude tracéologique aussi poussée que le permettait son bon état de conservation (Mélin, 2011; 2012). Celui de Petosse pourrait bénéficier d'une étude tout aussi approfondie, et permettre ensuite une comparaison des chaînes opératoires mises en œuvre pour chacun de ces ensembles, afin d'aborder la question des traditions techniques. Le processus de fabrication des haches de type vendéen pourrait être exploré en particulier. Peut-on reconnaître une même chaîne opératoire, des outils employés en post-fonderie similaires? Est-il possible de retrouver des moules communs entre ces deux dépôts, comme peuvent le suggérer les morphologies ressemblantes de différentes haches entre elles?

9. Dépôt au sujet duquel nous avons récemment fait un point concernant la localisation actuelle des haches : Mélin, 2015. 


\begin{tabular}{|c|c|c|}
\hline Objet & $\mathrm{n}^{\circ}$ inventaire musée Dobrée & dessin dans Breuil, 1902b \\
\hline Hache à rebords & 930.1 .222 & $\mathrm{n}^{\circ} 8$ \\
\hline Hache à rebords & 930.1 .223 & $\mathrm{n}^{\circ} 12$ \\
\hline Hache à rebords & 930.1 .225 & $\mathrm{n}^{\circ} 11$ \\
\hline Hache à rebords & 930.1 .226 & $\mathrm{n}^{\circ} 7$ \\
\hline Hache à rebords & 930.1 .227 & $n^{\circ} 6$ \\
\hline Hache à rebords & 930.1 .229 & $*$ \\
\hline Hache à rebords & 930.1 .230 & * \\
\hline Hache à talon & 930.1 .1024 & $\mathrm{n}^{\circ} 17 ?$ \\
\hline Hache à rebords, miniature & 56.2280 & * \\
\hline Hache à rebords, miniature & 56.2281 & * \\
\hline Hache à rebords, miniature & 56.2282 & * \\
\hline Hache à rebords & 56.2283 & * \\
\hline Hache à rebords & 56.2284 & * \\
\hline Hache à rebords & 56.2285 & * \\
\hline Hache à rebords & 56.2286 & * \\
\hline Hache à rebords & 56.2287 & * \\
\hline Hache à rebords & 56.2288 & * \\
\hline Hache à rebords & 56.2289 & $*$ \\
\hline Hache à rebords & 56.2290 & * \\
\hline Hache à rebords & 56.2291 & * \\
\hline Hache à rebords & 56.2292 & * \\
\hline Hache à rebords & 56.2293 & * \\
\hline Hache à rebords & 56.2294 & * \\
\hline Hache à rebords & 56.2295 & * \\
\hline Hache à rebords & 56.2296 & * \\
\hline Hache à rebords & 56.2297 & $*$ \\
\hline Hache à rebords & 56.2298 & * \\
\hline Hache à rebords & 56.2299 & * \\
\hline Hache à rebords & 56.2300 & * \\
\hline Hache à rebords & 56.2301 & * \\
\hline Hache à rebords & 56.2308 & * \\
\hline Hache à talon & 56.2311 & $\mathrm{n}^{\circ} 14$ \\
\hline Hache à talon & 56.2312 & $\mathrm{n}^{\circ} 16$ \\
\hline Hache à rebords et à talon & 56.2313 & $\mathrm{n}^{\circ} 13$ \\
\hline Hache à talon & 56.2315 & * \\
\hline Hache à talon & 56.2316 & $\mathrm{n}^{\circ} 15$ \\
\hline Hache à rebords & anc. coll de Fontaines & $\mathrm{n}^{\circ} 10$ \\
\hline Hache à talon & anc. coll de Fontaines & * \\
\hline Hache à rebords & anc. coll. Chauvet & $*$ \\
\hline
\end{tabular}

Tableau 2 : Liste des haches probablement issues du dépôt de Petosse, d'après nos recherches dans les réserves du musée Dobrée. Trenteneuf haches sont ainsi rassemblées : on est encore loin de la soixantaine déclarée par O. de Rochebrune.

Table 2: List of the axes that probably come from the Petosse hoard after preliminary research in the collections of the archaeological museum of Nantes. Thirty-nine axes have thus been identified still far from the sixty or so initially described by $O$. de Rochebrune. 


\section{Bibliographie}

Blanchet J.-C., Mordant C., 1987 - « Les premières haches à rebords et à butée dans le Bassin parisien et le Nord de la France ", dans Blanchet J.-C. (dir), Les relations entre le continent et les îles Britanniques à l'âge du Bronze, actes du colloque de Lille, 22e Congrès préhistorique de France (2-7 septembre 1984), Amiens, Revue archéologique de Picardie, p. 89-118.

Breuil H., 1902a - "Cachette de Saint-Étienne de Brillouet ", Revue archéologique, t. XL, p. 34-40.

Breuil H., 1902b - "Quelques haches ornées découvertes dans l'Ouest de la France ", Montauban (Association française pour l'avancée des sciences, t. XXXI), p. 926-931.

Fillon B., Rochebrune O. (DE), 1887 - Poitou et Vendée. Études historiques et artistiques, Niort, Clouzot, $2 \mathrm{t}$.

Gomez de Soto J., 1995 - Le Bronze moyen en Occident : la culture des Duffaits et la civilisation des Tumulus, Paris, Picard, 375 p.

Le Quellec J.-L., 1989 - "Précisions sur quelques haches en bronze du Centre-Ouest, inédites ou peu connues ", Bulletin de la Société préhistorique française, 86, 5, p. 134-136.

Lagarde-Cardona C., 2012 - Production métallique en Aquitaine à l'âge du Bronze moyen. Techniques, usages et circulation, Bordeaux, Ausonius, 420 p. (Scripta Antiqua, 39).

MÉLIN M., 2011 - Étude typologique et tracéologique des haches du dépôt daté du Bronze moyen 2 de Mouilleron-en-Pareds (Vendée), rapport d'étude, Nantes, SRA Pays de la Loire, 77 p.
MÉLIN M., 2012 - «Un nouveau dépôt vendéen du Bronze moyen 2 à Mouilleron-en-Pareds : présentation liminaire de son étude typologique et tracéologique ", Bulletin du Groupe vendéen d'études préhistoriques, 48, p. 1-17.

Mélin M., 2014 - «Le dépôt du Bronze moyen 2 de Mouilleronen-Pareds (Vendée) et les haches à rebords de type vendéen ", Revue archéologique de l'Ouest, 31, p. 83-106.

MÉLIN M., 2015 - «Visite des collections archéologiques du musée de Noirmoutier : investigation sur quelques dépôts métalliques du Bronze moyen 2 de Vendée et Loire-Atlantique », Bulletin du Groupe vendéen d'études préhistoriques, 51, p. 42-50.

Parpoil C., 2007 - Patrimonisation d'une collection : le legs de la collection Raoul de Rochebrune (1849-1924) au musée Dobrée, Nantes (1930), thèse de doctorat, université de Montpellier.

Pautreau J.-P., 1979 - Le Chalcolithique et l'âge du Bronze en Poitou (Vendée, Deux-Sèvres, Vienne), Poitiers, CAEP, 2 t., $432 \mathrm{p}$.

Poissonnier B., 1997 - La Vendée préhistorique, La Crèche, Geste éditions, $367 \mathrm{p}$.

Rochebrune O. (DE), 1880 - Les armes depuis l'âge celtique jusqu'au XVIII s. - Collections de Terre Neuve appartenant à Octave de Rochebrune, Niort, Clouzot.

Rochebrune O. (DE), 1901 - «Découvertes de haches celtiques et d'un torque gaulois à Velluire ", Revue du Bas-Poitou, $14^{\mathrm{e}}$ année, p. 277-283. 